\title{
DE LA COMÉDIE HUMAINE À LA COMÉDIE DIVINE: POLITIQUE, PHILOSOPHIE ET ICONOGRAPHIE: LES VECTEURS DE LA SATIRE DES DIEUX CHEZ LUCIEN
}

Isabelle Gassino*

Université de Rouen-ERIAC

\begin{abstract}
RESUMO: A questão da representação dos deuses em Luciano é vasta e complexa; este artigo tem por objetivo unicamente examinar as modalidades e os objetivos desta representação, mais do que as convicções religiosas do autor. O ponto de partida é o seguinte: quando Luciano evoca uma reunião de deuses, recorre a um vocabulário político que parece empregado sistematicamente demais para ser o simples fruto do acaso. Tratar-se-á de tentar compreender as razões desta escolha e, a partir disso, de contribuir para a compreensão dos procedimentos da sátira em Luciano.
\end{abstract}

PALAVRAS-CHAVE: sátira; comédia; deuses; filósofos; vida política.

es textes de Lucien mettant en scène les dieux grecs, on retient le plus souvent le ton satirique et l'image ridicule que l'auteur donne des immortels. De Photius aux humanistes de la Renaissance, on a vu en Lucien un érudit dont la lecture avait le mérite de détourner du paganisme, et, au Siècle des Lumières, Voltaire a pu faire de lui un porte-parole de sa propre critique de l'Eglise catholique. Les études ne manquent pas qui s'interrogent sur la religion de Lucien, sur son éventuel athéisme, ainsi que sur les liens qu'il peut entretenir avec les différentes écoles philosophiques, le but commun de ces études étant de mettre en lumière les orientations de la pensée de Lucien.

Bien qu'il soit, à notre avis, impossible de définir précisément les convictions religieuses ou athées de Lucien, nous voudrions à notre

^ isabelle.gassino@univ-rouen.fr 
tour reprendre la question vaste et complexe de la représentation des dieux $^{1}$ chez lui, en nous focalisant sur les modalités et les buts de cette représentation: a-t-on affaire à une satire gratuite, simple amusement d'un auteur souhaitant distraire son public, ${ }^{2}$ mais peu intéressé par l'observation de l'actualité, ${ }^{3}$ ou bien les dieux sont-ils le vecteur d'une critique plus structurée et plus profonde?

Nous partirons de la conclusion que donne O. Karavas à l'un de ses récents articles ${ }^{4}$ en disant que Lucien n'est pas un nihiliste et que "sa satire religieuse montre toute une recherche profonde sur le sujet". Nous proposerons une relecture de deux textes complémentaires: l'Assemblée des dieux et le début du Zeus tragédien, qui montrent tous deux les dieux réunis pour débattre de leurs problèmes communs. Notre fil conducteur sera le suivant: au-delà des moqueries récurrentes sur les dieux, évidemment destinées à amuser l'auditoire, les textes en question manifestent-ils une pensée cohérente sur les problèmes religieux, ou bien même, le cas échéant, sur des questions d'un autre ordre? En d'autres termes, Lucien peut-il être sérieux quand il fait ce type de satire, ou bien n'est-il définitivement qu'un amuseur public? ${ }^{5}$

\footnotetext{
${ }^{1}$ Nous n'aborderons pas la question de la représentation des dieux ne faisant pas l'objet d'un culte en Grèce; nous laisserons notamment de côté le cas particulier posé par le traité Sur la déesse syrienne, à propos duquel on pourra consulter l'étude exhaustive et approfondie de J. L. Lightfoot (Lucian: On the Syrian goddess. Oxford: Oxford University Press, 2003).

${ }^{2}$ Cf. Caster, op. cit., p. 366: "Son attitude est celle d'un homme du monde qui se moque et n'a jamais eu de plan d'ensemble".

${ }^{3}$ Cf. Bompaire, op. cit., p. 490-499; p. 495: "À quelques exceptions près, les développements religieux sont une illustration privilégiée de l'inactualité de Lucien". ${ }^{4}$ Cf. Karavas, op. cit., p. 137-144.

${ }^{5}$ Les traductions de Lucien données ici nous sont propres. Le texte grec cité est celui de l'édition Bompaire (C.U.F., Belles Lettres) pour les opuscules 1 à 29; pour les autres, celui de l'édition MacLeod (Oxford Classical Texts). Pour les auteurs autres que Lucien, les textes et traductions utilisés sont ceux de la C.U.F., quand ils existent; pour Philostrate et Athénée, nous avons eu recours à l'édition Loeb, et à l'édition Teubner pour Pollux; pour ces trois auteurs, la traduction donnée a été faite par nos soins.

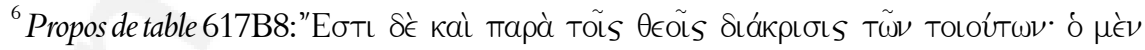

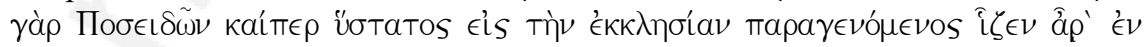

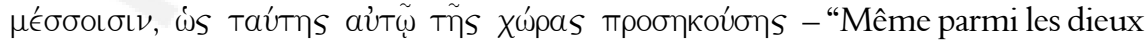
se fait une distinction pour ces sortes de préséances: ainsi Poséidon, quoique arrivé le dernier à l'assemblée, s'assied au milieu d'eux, parce que cette place lui revient".
} 


\section{Les assemblées des dieux: un reflet de la vie politique athénienne?}

Lorsque Lucien met en scène une réunion de dieux, il a recours à un vocabulaire politique dont la présence paraît trop affirmée pour être totalement fortuite: nous examinerons quelle est sa raison d'être et les liens qui peuvent exister entre la satire des dieux et la représentation de la vie politique athénienne de l'époque de Lucien.

\section{I.1. Monde des dieux et cité athénienne: un vocabulaire commun}

Un trait de vocabulaire frappe immédiatement: lorsque les dieux

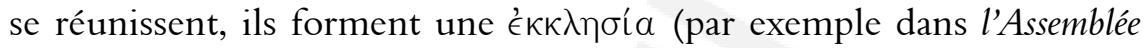

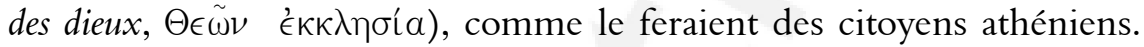
En effet, il n'y a que l'assemblée du peuple athénien à s'appeler

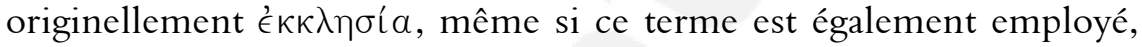
par extension, pour désigner des assemblées non athéniennes: en effet, on le trouve dans la Politique d'Aristote $(3,14,4)$ pour parler des assemblées chez Homère (alors que le mot homérique est ảyopń).

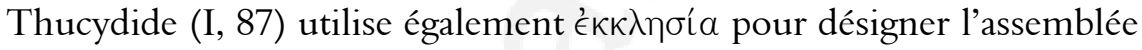

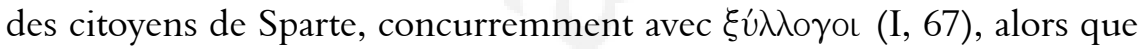
le nom propre à cette institution est, comme on le sait, $\dot{a} \pi \epsilon \lambda \lambda \dot{a}$.

Toutefois, ce mot à lui seul ne serait pas suffisant pour attester une comparaison entre l'univers des dieux et la cité athénienne. En

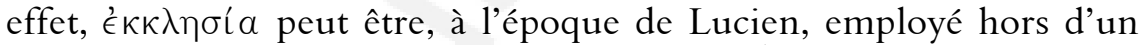
contexte politique: on le trouve chez Plutarque ${ }^{6}$ pour désigner, comme chez Lucien, une réunion des dieux.

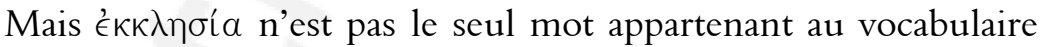
politique qui soit utilisé pour parler des dieux. Voyons par exemple l'ordre du jour de l'assemblée des dieux (Assemblée des dieux 3):

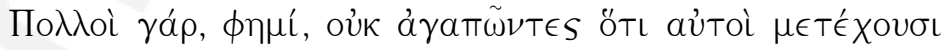

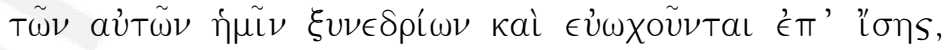

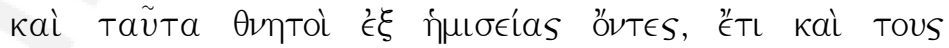

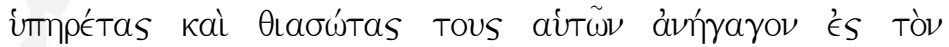

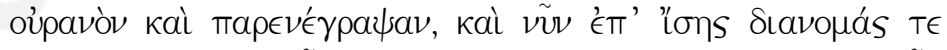

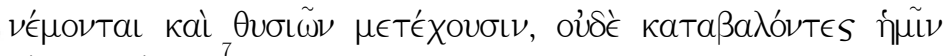

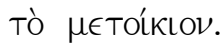

\footnotetext{
7 "Beaucoup d'entre nous, dis-je, non contents de participer aux mêmes assemblées que nous et d'avoir, dans nos banquets, des parts égales aux nôtres, et cela tout en
} 
Outre le mot $\xi v \nu \epsilon ́ \delta \rho เ \nu$, qui désigne une assemblée politique

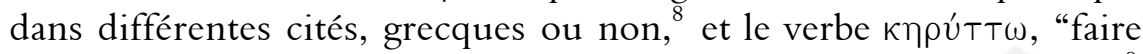
une proclamation", qui, chez notre auteur, a toujours pour sujet Hermès ${ }^{9}$ (ce qui correspond bien à sa fonction de héraut des dieux) on relève les mots $\mu \in$ Tó́kıov, désignant la taxe spécifique payée par les métèques, ${ }^{10}$ et le verbe $\pi a \rho \epsilon \gamma \gamma \rho a ́ \phi \omega$, littéralement "inscrire à côté", en particulier "inscrire un nom sur une liste", couramment utilisé dès l'époque classique pour décrire l'action d'ajouter frauduleusement un nom sur

étant à moitié mortels, ont, de plus, amené dans le ciel et fait inscrire frauduleusement sur la liste des dieux leurs serviteurs et les membres de leur thiase, et maintenant ceux-ci ont une part égale à la nôtre dans les distributions et les sacrifices, sans même nous verser la taxe des métèques".

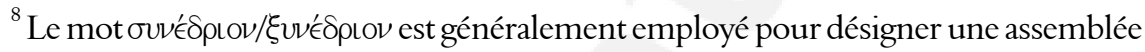
politique, et cela dans des contextes variés: sont ainsi nommés le conseil des Syracusains (Xénophon, Helléniques, I, 1,31), l'amphictyonie delphique (ibid., VII, 39) et le sénat romain (Polybe, Histoires, I, 11, 1). Un passage de Platon (Protagoras 317d) fait exception dans la mesure où le mot renvoie à l'assemblée constituée par Protagoras et Socrate et leurs auditeurs; il ne s'agit évidemment pas d'une réunion politique, mais le terme peut être employé par métaphore en référence à une assemblée publique. En outre, le terme de $\sigma v \nu \in \delta \rho i ́ \alpha$, lui, peut aussi s'employer dans un contexte amical et désigner, de manière générale, le fait d'être ensemble. Les mots de cette famille ont donc un sens beaucoup plus large que le strict usage politique. Dans notre passage également, $\xi v v e ́ \delta \rho ı \nu$ paraît plus large de sens

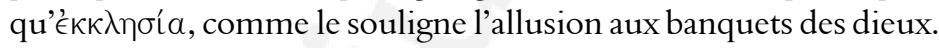

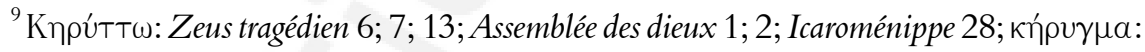
Zeus tragédien 6 (trois occurrences du mot); Assemblée des dieux 1.

${ }^{10}$ On sait qu'il a existé des métèques dans d'autres cités qu'Athènes, mais on ne sait pratiquement rien d'eux avant l'époque hellénistique. (cf. Gauthier, op. cit.) Nous ne saurions donc assurer, sur ce point, que Lucien décrit une réalité spécifiquement athénienne. On ne peut pas davantage émettre d'hypothèse sur la date à laquelle cette allusion peut renvoyer: si le statut des métèques évolue à l'époque hellénistique, le paiement du métoikion est resté en vigueur très longtemps, au point que l'acquittement de cette taxe sert encore, au IIe s. ap. J.-C., à définir le métèque.

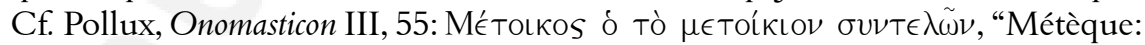
celui qui acquitte le métoikion". La mention du thiase, qui introduit une discussion sur la présence de Dionysos dans l'Olympe, ne peut pas davantage nous fournir de point d'ancrage temporel, ces associations religieuses - et les cortèges dionysiaques qu'elles pouvaient former - existant depuis la fin de l'époque archaïque au moins. Cf. Freyburger, Freyburger et Tautil, op. cit., p. 61. 
la liste des citoyens. ${ }^{11}$ La réunion des dieux prend donc bel et bien la même forme qu'une assemblée politique athénienne, ce qui est tout à fait confirmé par la fin du texte, puisque les dieux rédigent, à l'issue de la réunion, un décret qui pastiche ceux que prenaient les cités grecques (Assemblée des dieux 14):

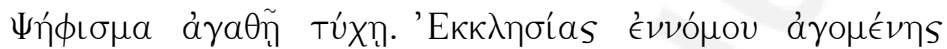

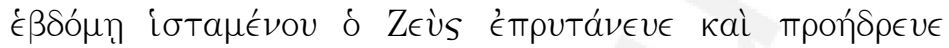

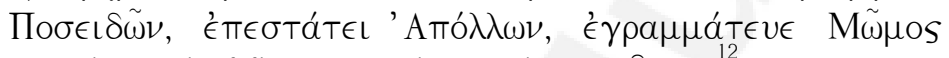

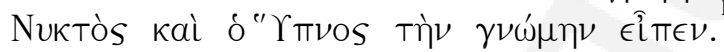

\section{I.2. Parallèles avec l'actualité du lle siècle après Jésus- Christ}

Outre des emprunts à un vocabulaire certes spécifique mais non lié à une époque déterminée, un parallèle a pu être fait entre ce texte et un événement qui eut lieu à Athènes sous le règne de Marc-Aurèle. En effet, si la révision des listes des dèmes et des phratries était un phénomène ancien, qui se produisait plus ou moins régulièrement depuis le milieu du Ve s. au moins, ${ }^{13}$ il se trouve aussi que la question de l'admission de nouveaux membres au sein d'une assemblée prestigieuse a été justement débattue à Athènes vers l'an 165, date à laquelle on peut estimer que le texte de Lucien a été composé. ${ }^{14}$ Nous nous appuierons ici sur une étude de James H. Oliver ${ }^{15}$ faisant suite à un article de Simone Follet. ${ }^{16}$ À Athènes au IIe s. ap. J.-C., la citoyenneté

\footnotetext{
${ }^{11}$ Eschine, Sur l'ambassade, 76 et 177 . Cet emploi se trouve également chez des

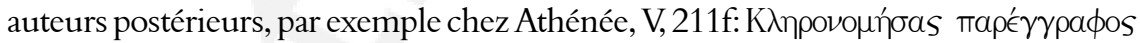

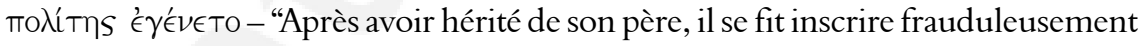
sur la liste des citoyens".

12 "Décret. À la bonne fortune! L'assemblée s'est réunie conformément au règlement, le sept du mois, avec Zeus pour prytane, Poséidon pour proèdre, Apollon pour épistate, Momos, fils de Nuit, pour secrétaire et le Sommeil a fait la proposition suivante".

${ }^{13}$ Plutarque, Vie de Périclès 37, 4: allusion à une distribution de blé aux citoyens d'Athènes qui fut faite vers 445 et donna lieu à une révision drastique des listes des dèmes et des phratries pour en ôter ceux qui s'y étaient fait inscrire frauduleusement.

${ }^{14}$ Cf. Schwartz, op. cit.

${ }^{15}$ Cf. Oliver, op. cit., 1980, p. 303-313.

${ }^{16}$ Cf. Follet, op. cit., p. 29-43.
} 
n'était plus un privilège parcimonieusement accordé; Athènes et Délos étaient des plaques tournantes du commerce en Méditerranée et attiraient de nombreux étrangers qui obtenaient souvent la citoyenneté athénienne, comme l'attestent les catalogues éphébiques de la cité. ${ }^{17}$ Par ailleurs, une nouvelle réforme de l'Aréopage est faite à cette époque. Les règles permettant d'être membre de cette prestigieuse assemblée ont en effet évolué au fil du temps: alors qu'au IVe s. avant J.-C., il fallait être issu de trois générations de citoyens athéniens, plus tard peut-être dès le IIe s. av. J.-C. - il a suffi d'être issu de trois générations d'hommes libres. ${ }^{18}$ Cette règle a encore été assouplie par Marc-Aurèle de manière que l'Aréopage devienne accessible à des petits-fils ou même à des fils d'esclaves; mais cette disposition est annulée en 165, date à partir de laquelle, pour être archonte, il faut à nouveau être issu de trois générations d'hommes libres. ${ }^{19}$

L'Assemblée des dieux traite, elle, du problème posé par l'admission, parmi les dieux, d'un grand nombre de divinités étrangères, qui rend l'Olympe bondé et invivable. S'agit-il là d'une transposition dans l'univers des dieux de ce qui se passait à Athènes à la même époque? J. H. Oliver conclut en ce sens. Toutefois, même si le rapprochement est très séduisant, on ne peut prouver de manière formelle et définitive que le texte de Lucien s'inspire directement des realia athéniennes de l'époque, même si le parallèle semble trop évident pour être le fruit du hasard.

Les problèmes de relations entre dieux et, en particulier, les questions de préséance sont également abordées dans le Zeus tragédien, où Lucien rend compte de la hiérarchie - sur laquelle nous reviendrons - existant entre immortels en employant les termes de pentacosiomédimnes, zeugites, thètes: ainsi, à Aphrodite qui se plaint de ne pas être admise aux places d'honneur, Hermès répond qu'Apollon est "lui aussi, assis parmi les zeugites" et conclut: "Contente-toi donc, toi aussi, de ne pas siéger au dernier rang des thètes". ${ }^{20}$ En revanche, le colosse de Rhodes pourrait prétendre dépasser les pentacosiomédimnes. ${ }^{21}$ On pourrait se contenter

\footnotetext{
${ }^{17}$ Cf. Follet, op. cit.

${ }^{18}$ Cf. Follet, op. cit.

${ }^{19}$ Cette règle est à nouveau modifiée en 174/ 175 par un édit de l'empereur. Cf. Oliver, op. cit., 1980.

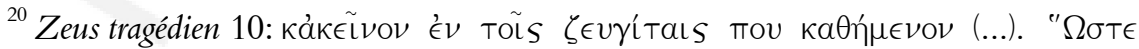

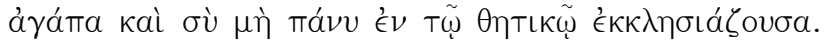

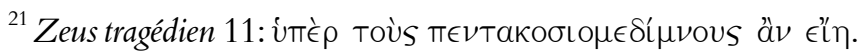


de voir là l'un des innombrables traits réputés prouver que Lucien est un nostalgique de l'Athènes démocratique et indépendante de l'époque classique; mais il se trouve que l'empereur Marc-Aurèle avait manifesté une volonté très claire de renouer avec les anciennes classes soloniennes, de sorte que, vers 175 ap. J.-C., on retrouve les différents niveaux d'éligibilité que l'on avait vers $470 \mathrm{av}$. J.-C.: les pentacosiomédimnes et les hippeis étaient éligibles à l'Aréopage et au Conseil; les zeugites l'étaient au Conseil et aux magistratures inférieures; quant aux thètes, ils n'étaient pas éligibles et ne pouvaient que prendre part aux assemblées du peuple. ${ }^{22} \mathrm{La}$ terminologie de Lucien peut donc renvoyer à la fois à une réalité ancienne tout en coïncidant avec un renouveau de l'emploi de ces dénominations.

Ici encore, il existe une ressemblance étonnante entre ce qu'écrit Lucien et les événements contemporains. Les nouveaux dieux se voient reprocher de ne même pas "payer la taxe des métèques"; ${ }^{23}$ or, nous avons vu que les personnes introduites dans l'Aréopage à la faveur de la réglementation assouplie, antérieure à Marc-Aurèle, étaient des descendants d'esclaves affranchis, lesquels, à l'époque classique, avaient un statut intermédiaire comparable, précisément, à celui des métèques. Lucien fait-il à dessein un parallèle entre ces hommes et les nouveaux dieux, ou ce rapprochement n'est-il que le fruit du hasard? Nous ne disposons pas d'élément qui permette de trancher la question, mais si hasard il y a, force est de constater qu'il fait bien les choses.

\section{I.3. Des dieux sujets ou des dieux reflets?}

Revenons brièvement sur un aspect de l'Assemblée des dieux: si l'on voit dans ce texte une allusion aux réformes de l'Aréopage voulues par Marc-Aurèle, le seul décalage introduit par Lucien résiderait dans la substitution, dans son texte, de l'ecclèsia à l'Aréopage; cela veut-il dire que Lucien transpose la scène dans une époque plus reculée, où l'ecclèsia était l'organe majeur du gouvernement d'Athènes - alors qu'au IIe s., c'était l'Aréopage qui remplissait cette fonction? On peut plutôt penser que Lucien opte simplement pour un terme plus général et plus neutre; comme on l'a vu, le mot "ecclèsia" était assez répandu, contrairement à celui d' "Aréopage". Lucien fait, selon toute vraisemblance, un rapprochement avec l'actualité politique de l'époque,

\footnotetext{
${ }^{22}$ Cf. Follet, op. cit.

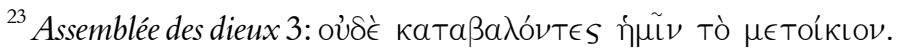


mais il ne fait pas de celle-ci une clef de compréhension de son texte. ${ }^{24}$ C'est là un élément très important, car il nous indique sur quoi porte l'accent du texte de Lucien: celui-ci n'est pas une sorte de restitution cryptée de la réalité de son temps, car dans ce cas Lucien aurait probablement fait des allusions beaucoup plus précises et directes, de manière que le lecteur saisisse à coup sûr l'allusion. Si, à ses yeux, l'important dans ce dialogue avait été de parler de la réforme de l'Aréopage, il n'aurait pas transposé la question à l'ecclèsia. Au contraire, ici, l'actualité politique est une toile de fond servant à mettre en scène un sujet plus important: les dieux et les contradictions auxquelles ils sont confrontés. Ce sont donc bien les dieux qui sont le sujet principal et l'allusion à l'actualité est le moyen, et non la fin, de la représentation.

Peut-on pousser le raisonnement jusqu'à voir dans la figure de Zeus une transposition de l'empereur Marc-Aurèle? ${ }^{25}$ Dans ce cas, les dieux seraient le vecteur d'une satire visant également, ou principalement, l'empereur. Un autre texte de Lucien au moins se prête à un tel rapprochement: il s'agit des Fêtes de Cronos, qui rapporte une requête adressée par les citoyens pauvres à Cronos, afin que les riches cessent leurs vexations envers les pauvres et en particulier pour qu'ils ne cherchent plus, par des subterfuges mesquins, à fuir les obligations que leur créent les fêtes du dieu. Cette requête est l'occasion d'un échange de lettres entre, d'une part, Lucien, porte-parole des pauvres, et Cronos, d'autre part entre Cronos et les riches.

On peut rapprocher ce texte d'une inscription trouvée à Athènes, sur l'Agora, dans les années $1930{ }^{26}$ Cette inscription est une réponse de l'empereur Marc-Aurèle à une requête des Athéniens, alors en conflit avec Hérode Atticus. Elle est datée de l'an 174/ 5, ${ }^{27}$ c'est-à-dire peu de temps avant le retour d'Hérode Atticus à Athènes après son exil. Elle se présente comme un édit destiné à régler un différend, mais en fait elle est adressée à des personnes précises, même si le document n'a jamais l'allure formelle d'une lettre. ${ }^{28}$ L'origine de la querelle est

\footnotetext{
${ }^{24}$ Comme le fait remarquer Jones, op. cit., ch. 4.

${ }^{25}$ C'est ce que fait Oliver, op. cit., 1980.

${ }^{26}$ Cf. Oliver, op. cit., 1970.

${ }^{27}$ Cf. Oliver, op.cit., 1970 , p. 35.

${ }^{28}$ Par exemple, Marc-Aurèle néglige d'employer le $\epsilon$T$\tau \cup \chi \epsilon \tilde{\imath} \epsilon \epsilon$ final qui clôt ordinairement les lettres.
} 
incertaine, comme l'indique Philostrate, ${ }^{29}$ mais il apparaît que les Athéniens demandaient justice à Marc-Aurèle de certaines brimades et contraintes qui leur étaient infligées par le puissant Hérode Atticus et ses affranchis. ${ }^{30}$

Nous relèverons plusieurs points communs à ces deux textes:

1) Il s'agit de réponses à des requêtes déposées auprès d'un personnage qui détient le pouvoir suprême ou est considéré comme tel par les plaignants; ce personnage, sans refuser d'intervenir, s'en remet aussi, pour une part, à la décision d'un tiers.

2) La plainte, dans les deux cas, est motivée par des injustices qui tournent à l'humiliation.

3) Le personnage dont on demande l'arbitrage souhaite avant tout réconcilier les deux parties, dont la désunion menace la cohésion sociale.

4) Dans le texte de Lucien, le rapprochement avec une situation politique est suggéré par l'emploi de certains termes (l'adjectif

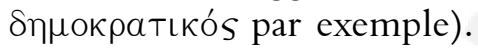

Le rapprochement avec la requête adressée à l'empereur offre ici encore une clef de lecture possible mais pas obligatoire: en effet, les adresses à l'empereur étaient fréquentes, de sorte que l'on ne saurait affirmer que Lucien fait précisément référence à l'affaire que nous avons évoquée. On peut également imaginer que Lucien fasse allusion à un mode de communication entre les citoyens et l'empereur plutôt qu'à un événement précis.

${ }^{29}$ Vie des Sophistes, 559-560: Philostrate présente comme "la cause la plus probable"

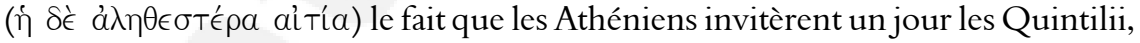
alors gouverneurs de Grèce, à une séance de l'ecclèsia et se plaignirent à eux du

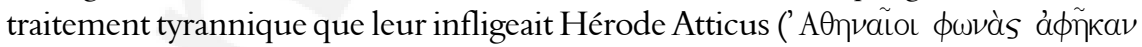

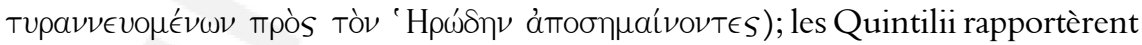
les faits à Marc-Aurèle, que certains Athéniens allèrent trouver personnellement en Pannonie, à Sirmium. Mais les causes des plaintes athéniennes ne sont pas rapportées par Philostrate. On peut supposer que l'affaire du testament d'Hérode Atticus n'y était pas totalement étrangère (\$549).

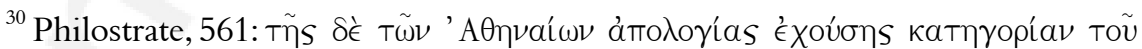

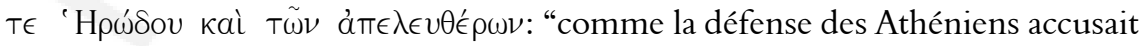
Hérode et ses affranchis"; sur cet épisode de l'histoire athénienne, voir aussi Graindor, op. cit., ch. VIII et, pour une mise à jour de ce dernier ouvrage, assortie d'un supplément archéologique, Tobin, op. cit. 
Bien évidemment, le rapprochement entre le monde politique athénien et les dieux n'a rien de nouveau à l'époque de Lucien: c'est en effet un procédé que l'on trouve, pour ne citer que ce seul exemple, dans les comédies d'Aristophane, où Périclès est représenté en Zeus. À l'instar du maître de l'Olympe, Périclès lance l'éclair et tonne quand on le mécontente, selon une métaphore bien connue, dont témoigne le surnom d'“Olympien" qui lui était dévolu; c'est le cas dans les Acharniens:

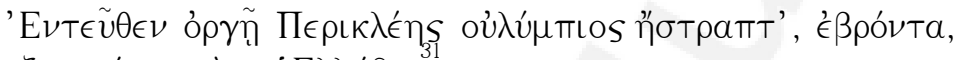

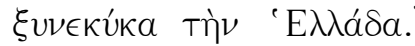

N'importe quel chef politique peut d'ailleurs se voir appliquer la même image: ainsi, le démagogue Cléon fait éclater "le tonnerre de ses discours". ${ }^{32}$

La nouveauté introduite par Lucien n'est pas dans l'image, mais dans l'inversion du rapport comparant/ comparé. Dans les deux exemples ci-dessus, en effet, ce sont les dieux qui constituent le comparant, et, même si l'image n'est pas univalente, même si elle est irrévérencieuse vis-à-vis des dieux mêmes, elle n'en fait pas moins appel à la notion d'une divinité dont la puissance impressionne les hommes. Ce n'est plus le cas chez Lucien, puisque les hommes s'adressent aux dieux en leur parlant d'égal à égal. ${ }^{33}$ Chez Lucien, au contraire, ce sont les dieux qui sont comparés aux hommes: le point de comparaison n'est plus le divin, mais l'humain, ce que peut résumer la remarque pleine de lassitude que fait Zeus à Asclépios et Héraclès (Dialogues des Dieux XV):

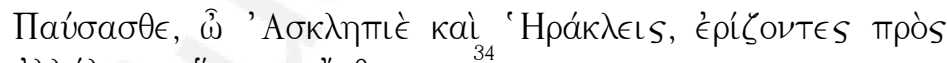

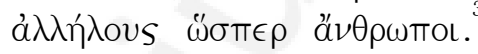

\section{Les dieux: plutôt de médiocres philosophes que de bons citoyens}

Même si le vocabulaire de la démocratie athénienne est utilisé pour décrire les réunions entre dieux, le comportement de Zeus à la fin de

\footnotetext{
${ }^{31}$ Vers 530 sqq.: "Là-dessus, colère de Périclès: l'Olympien lance l'éclair, fait gronder son tonnerre, bouleverse l'Hellade".

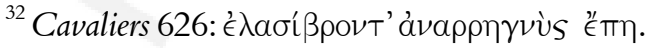

${ }^{33}$ Dans le Zeus confondu notamment.

34 "Asclépios et Héraclès, arrêtez de vous chamailler comme des humains".
} 
l'Assemblée des dieux (\$19) semble bien peu démocratique, puisqu'il finit par abandonner l'idée d'organiser un vote sur le décret qui vient d'être rédigé:

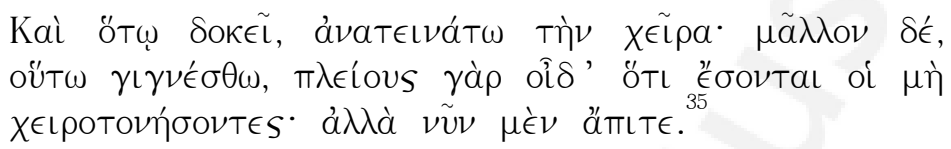

Zeus se trouve pris dans une contradiction insurmontable: comme il y a dans l'assemblée de nombreux dieux qui n'ont aucun intérêt à ce que ce décret soit adopté, il n'est pas possible de suivre les procédures habituelles et de se débarrasser légalement des gêneurs. C'est moins son comportement anti-démocratique que l'incohérence de celui-ci et l'impuissance qu'elle manifeste qui sont visés par la satire.

D'autre part, il arrive qu'un terme appartenant au vocabulaire politique ait un sens plus large: ainsi, lorsqu'il est question de $\pi \rho \circ \epsilon \delta \rho i ́ a$, le mot ne renvoie pas nécessairement à la fonction politique qu'il pouvait désigner dans l'Athènes classique, à savoir, la présidence de la séance. Le plus souvent, ${ }^{36}$ il signifie seulement "être assis au premier rang". ${ }^{37}$ D'ailleurs, même si ce terme revient à plusieurs reprises, ${ }^{38}$ il est employé concurremment avec le verbe $\pi \rho \alpha_{\alpha} \theta^{\prime} \zeta \omega^{39}$ qui, lui, n'implique pas de fonction politique. Il renvoie simplement au fait d'être assis au premier rang, ce qui était en soi un honneur. Ce que les dieux cherchent à obtenir, ce ne sont pas des responsabilités, mais simplement une marque de déférence qui flatte leur amour-propre et leur sens du confort - on peut supposer, à en juger par l'architecture des théâtres grecs et autres lieux d'assemblée, que le premier rang de sièges pouvait être pourvu d'un dossier - autrement dit une "bonne place". C'est le verbe

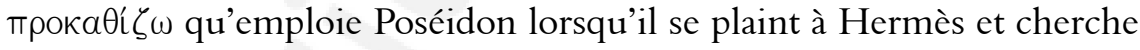
à obtenir la reconnaissance de sa supériorité sur Anubis par le biais d'une place honorifique (Zeus tragédien 9):

\footnotetext{
35 "Que ceux qui approuvent ce décret lèvent la main; ou plutôt non, qu'il soit appliqué sans autre formalité: car, je le sais, une majorité votera contre. Allons, partez à présent”.

${ }^{36}$ Sauf dans Assemblée des dieux 14, cité note 12.

${ }^{37}$ Cf. Delz, op. cit., ch. IV, p. 45.

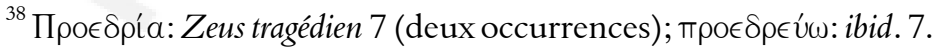

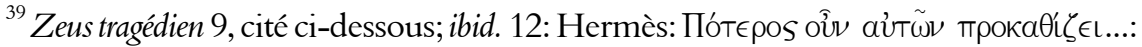
"Lequel des deux [Dionysos ou Héraclès] aura la préséance".
} 


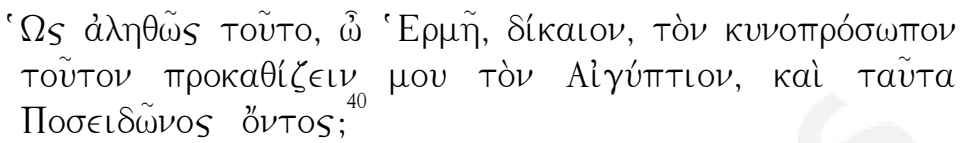

Lucien mêle également au vocabulaire politique des éléments étrangers à celui-ci: ainsi, au début de l'Assemblée des dieux, les termes

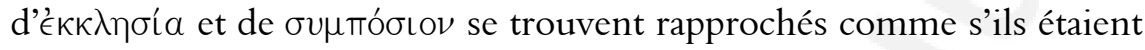
équivalents (Assemblée des dieux 1):

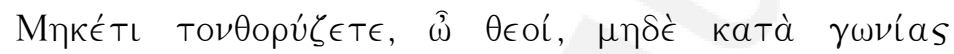

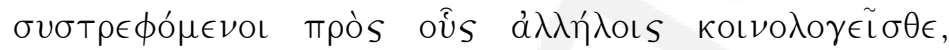

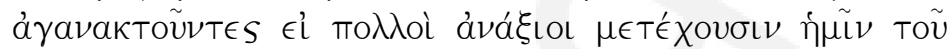

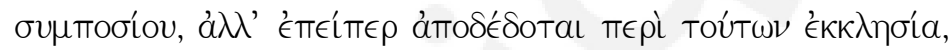

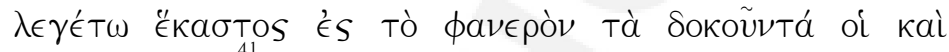

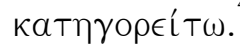

Ce qu'il y a d'étonnant, ici, c'est l'association d'une assemblée et d'un banquet. Pour prendre un terme de comparaison, chez Homère, où l'on trouve également des assemblées de dieux, jamais banquet et réunion d'un conseil ne sont confondus. ${ }^{42}$ Dans le passage de Lucien qui

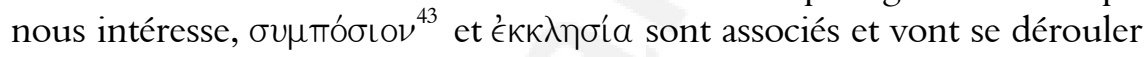
en même temps. Ce n'est toutefois pas systématiquement le cas, puisque le Zeus tragédien ne fait aucune mention d'un $\sigma v \mu$ Tó $\sigma \mathrm{o \nu}$, mais précisément, cette séparation est tout à fait inhabituelle, comme le montre la réaction des dieux, qui sont étonnés de l'absence d'ambroisie et de victimes et réclament ce qu'ils estiment leur être dû (Zeus tragédien 13):

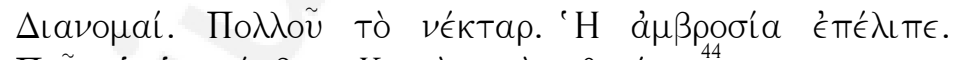

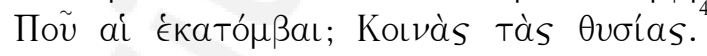

\footnotetext{
40 "Non mais vraiment, Hermès, est-il juste que cette face de chien, cet Egyptien, soit assis devant moi? Je suis Poséidon, tout de même!"

41 "Arrêtez de ronchonner, dieux, arrêtez de parler entre vous en vous regroupant dans votre coin et de vous indigner de ce que de nombreux dieux prennent part à notre banquet alors qu'ils n'y ont pas droit: puisqu'une assemblée a été convoquée à ce sujet, que chacun dise clairement ce qu'il pense et formule ses griefs".

${ }^{42}$ Iliade VIII, 1-40; XX,1-30; Odyssée I, 11-95; V, 1-42.

${ }^{43}$ On trouve également employé, un peu plus loin (\$3), le verbe $\epsilon \dot{\omega} \omega X 0 \tilde{u} \mu a$, "se régaler".

44 "Distribution des parts! Le nectar est cher! Il n'y a plus d'ambroisie. Où sont les hécatombes? Les sacrifices sont pour tout le monde!"
} 
Lucien procède ainsi à un brouillage des pistes, à un mélange des genres analogue à celui qu'il fait à propos des philosophes et qui a été analysé par Luciana Romeri: ${ }^{45}$ celle-ci montre que, dans les banquets philosophiques "sérieux", comme celui de Platon, quand il y a discours philosophique, il y a silence autour de la nourriture. On ignore absolument ce qui est servi aux convives; quand il y a nourriture, il n'y a pas parole, et inversement. Dans le Banquet ou les Lapithes de Lucien, au contraire, les convives, pourtant des philosophes réputés respectables, ne se soucient guère d'échanger de savants discours: ils ont pour seul désir de profiter des plaisirs de la table et de s'emparer du plus grand nombre de mets, même s'ils doivent, pour cela, en venir aux mains; ${ }^{46}$ Lucien met en scène une grande quantité de plats et de vin, et ridiculise toute tentative de parole philosophique. De la même façon, dans le cas des dieux, la tentative de débat sérieux est mise à mal par la confusion entre réunion avec débat et réunion avec repas.

De même encore, les dieux de Lucien se disputent pour des questions de préséance exactement comme le font les philosophes ridicules du Banquet, qui prétendent tous aux places d'honneur ( $(9)$ :

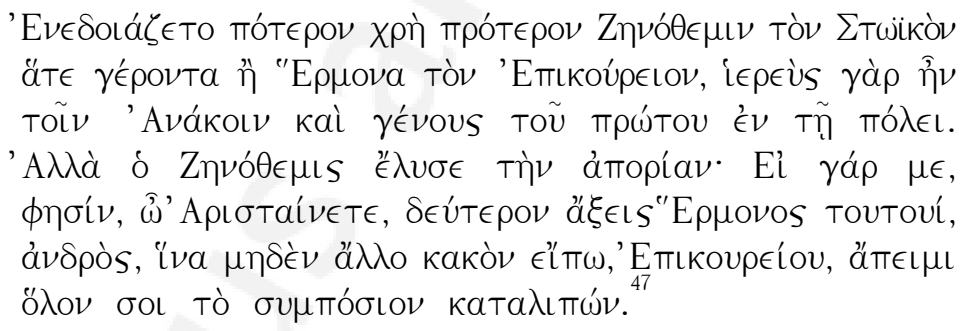

Ridicules, ces philosophes le sont d'autant plus qu'Hermon prétend à la place d'honneur comme prêtre des Dioscures, ce qui est un comble pour un épicurien, censé être critique vis-à-vis de la religion traditionnelle!

Autre point commun entre les dieux et les philosophes: la structure de la scène de convocation des dieux reproduit celle de l'appel des faux philosophes à la fin du Pêcheur. Dans ce dialogue, en effet, le

${ }^{45}$ Cf. Romeri, op. cit., p. 647-655.

${ }^{46}$ Voir notamment $\$ 42$.

47 "On hésitait: fallait-il donner la préséance au stoïcien Zénothémis eu égard à son grand âge ou à l'épicurien Hermon (car il était prêtre des Dioscures et appartenait à la première famille de la cité)? Mais Zénothémis résolut le problème en disant: 'Si tu me places, Aristénète, plus bas que cet Hermon, que cet épicurien - pour ne pas dire pire - je m'en vais en laissant tomber tout ton banquet'”. 
Syllogisme commence par appeler les philosophes en utilisant des formules ordinaires auxquelles personne ne prête attention; aussitôt après intervient Parrhèsiadès, alias Lucien, ${ }^{48}$ qui emploie une méthode autrement plus efficace (Pêcheur 41-42):

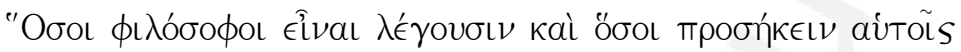

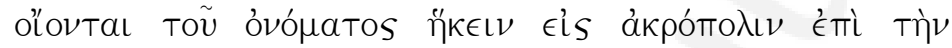

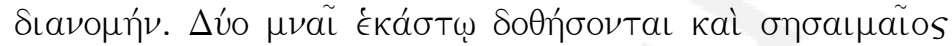

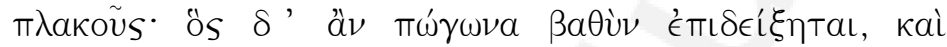

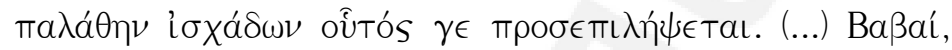

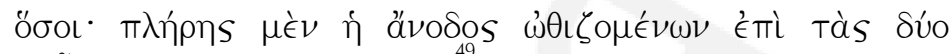
$\mu \nu \tilde{a} s$, is

De même, dans le Zeus tragédien, Hermès commence par faire une proclamation ordinaire, en prose, qui n'attire aucun des dieux à l'assemblée; sur les injonctions de Zeus, il fait une nouvelle tentative, en vers cette fois, et s'inspire de ce passage du chant XX de l'Iliade (5-9) pour composer quelques hexamètres mentionnant des hécatombes:

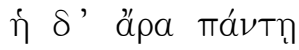

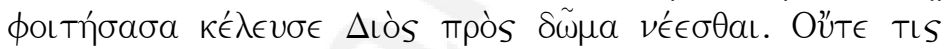

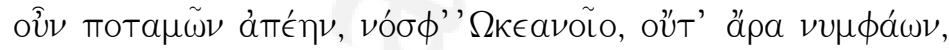

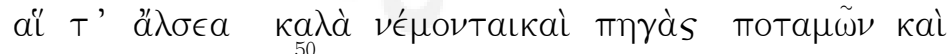

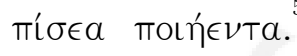

Aussitôt les dieux, par l'odeur des victimes alléchés, arrivent en foule (Zeus tragédien 6-7):

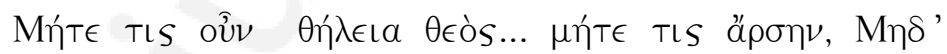

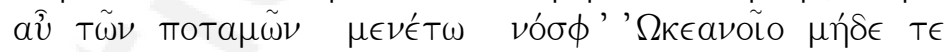

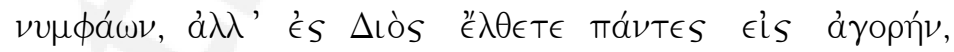

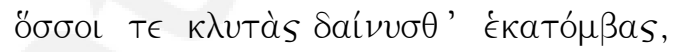

${ }^{48}$ Cf. Dubel, op. cit., p. 19-26.

49 "Que tous ceux qui se disent philosophes et tous ceux qui pensent mériter ce nom viennent à l'acropole pour avoir part à la distribution. On donnera à chacun deux mines et une galette de sésame, et quiconque arborera une barbe profonde recevra en outre un chapelet de figues sèches. (...) Oh la la! Que de monde! La montée s'est remplie de gens qui se poussent pour avoir les deux mines, sitôt qu'ils en ont entendu parler".

${ }^{50}$ Il. XX, 5-9: "Elle va donc de tous côtés leur porter l'ordre de se rendre au palais de Zeus. Pas un des fleuves ne manque - excepté Océan - pas une des nymphes habitant les bosquets charmants, les ondes des fleuves ou les prés herbus”. 


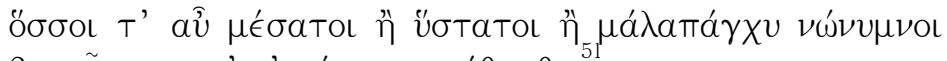

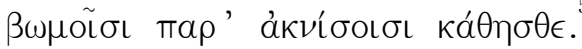

Comme dans le Pêcheur, l'efficacité de la nouvelle méthode est immédiate, ainsi que Zeus le constate (Zeus tragédien 7):

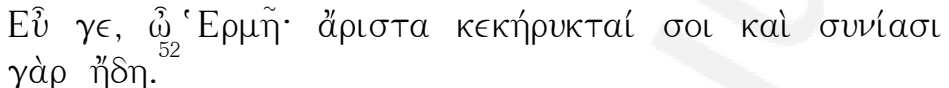

Le ressort comique est le même dans les deux passages: c'est à l'évocation d'une rétribution matérielle que réagissent les uns et les autres, de sorte que les dieux apparaissent comme des charlatans, tout comme les faux philosophes. La satire est toutefois plus modérée à l'égard des dieux, dans la mesure où ceux-ci ne sont pas attirés par l'argent. Les dieux, eux, sont mus par une gourmandise doublée d'une avidité de marques d'honneurs - passions finalement bien humaines: des hommes, ils n'ont donc pas seulement l'apparence, mais aussi les travers moraux.

Un autre trait de vocabulaire résonne étrangement dans le contexte d'une assemblée politique: c'est le mot $\sigma \kappa e ́ \psi \downarrow \iota s$, qu'Hermès emploie pour ouvrir la séance et indiquer l'ordre du jour (Assemblée des dieux 1):

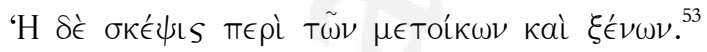

Très courte phrase, qui n'en est pas moins délicate à traduire, car le terme de $\sigma \kappa \in ́ \psi \downarrow \iota s$ n'appartient pas au registre politique classique: dans

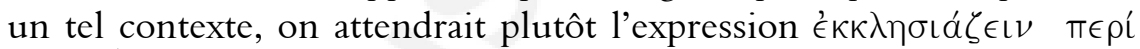

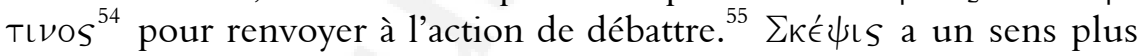

\footnotetext{
51 “Qu'aucun dieu, femelle ou mâle, qu'aucun fleuve même, à part l'Océan, qu'aucune nymphe ne reste sans venir. Non, mais venez tous chez Zeus, à l'assemblée, vous qui partagez d'illustres hécatombes, et vous, dieux de la classe intermédiaire, de la dernière classe, ou complètement anonymes qui siégez près d'autels n'exhalant aucun fumet”.

52 "Bien, Hermès; tu as fait une excellente proclamation, et les voilà déjà qui se rassemblent".

53 "La question porte sur les métèques et sur les étrangers".

${ }^{54}$ Cf. Thucydide, 7, 2, 2; Aristophane, Thesmophories, 84-85; Isocrate, Surla paix, 2.

${ }^{55}$ Il est vrai que, dans l'ensemble, la terminologie politique de Lucien n'est pas parfaitement homogène et qu'elle ne coïncide pas toujours avec la terminologie athénienne classique: ces particularités peuvent être le reflet d'influences d'Asie Mineure - où Lucien a voyagé et reçu sa première éducation rhétorique notamment des cités de Magnésie du Méandre, Tarse, Antioche (cf. Householder,
} 
précis et plus spécialisé que le verbe $\sigma \kappa \epsilon ́ \pi T o \mu a l$, qui est très courant chez tous les auteurs de l'époque classique; le substantif est principalement employé par les philosophes et désigne la réflexion, la recherche approfondie sur une question: ce sens remonte à Platon au moins. ${ }^{56}$ Ce n'est qu'exceptionnellement, et à une époque tardive, qu'il renvoie aux délibérations d'une assemblée, ${ }^{57}$ mais il ne figure pas dans les inscriptions dont Lucien a pu s'inspirer, pas plus que chez Éphore, par qui il a pu avoir connaissance de ces décrets. ${ }^{58}$ C'est donc vraisemblablement pour son appartenance au vocabulaire philosophique que Lucien a choisi ce mot.

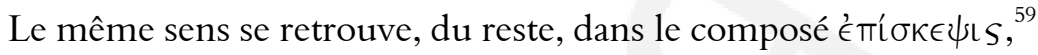
utilisé dans le Zeus tragédien 5, lorsqu'Hermès “affirme qu'il faut réunir une assemblée et s'en remettre, sur cette question, au jugement de tous

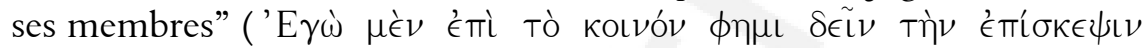

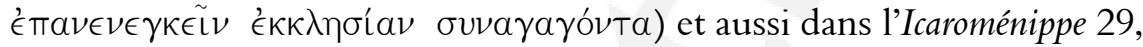
où l'on trouve le composé $\delta ı \alpha ́ \sigma \kappa \in \psi \iota s$. Zeus s'adresse aux dieux réunis

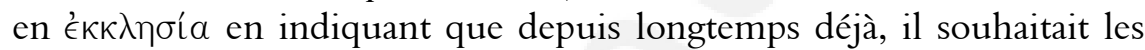
entretenir des philosophes et qu'il a jugé, avec l'arrivée de Ménippe,

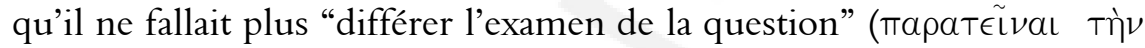
$\delta\llcorner a ́ \sigma \kappa \in \psi \nu \nu)$.

op. cit., p. 199-216); elles peuvent également être dues à des emprunts à des formules peu connues, mais parfaitement attiques, que l'on peut relever chez Thucydide d'une part, dans des décrets athéniens d'époque impériale, d'autre part.

${ }^{56}$ On relève plusieurs dizaines d'occurrences de ce mot chez Aristote (73) ou Platon (67), mais aucune référence chez Isocrate et une seule dans tout le corpus démosthénien, où il s'applique à tout examen préalable à une décision, aussi bien

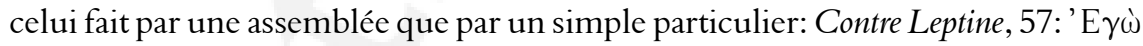

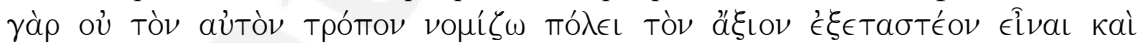

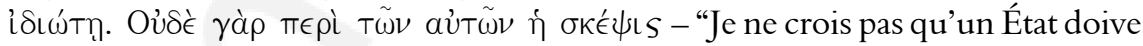
juger d'après les mêmes règles qu'un particulier. Ce n'est pas sur les mêmes points que porte l'examen".

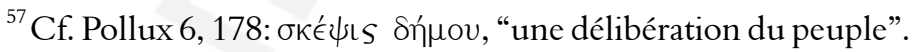

${ }^{58}$ Il est possible, comme le suggère Householder (op. cit.), que Lucien reprenne l'aspect formel des décrets rapportés par l'historien Éphore ou de ceux de cités de la mer l'Égée ou d'Asie mineure pour rédiger les lois de l'Assemblée des dieux (14-18), mais rien ne prouve qu'il le fasse dans l'intention d'imiter un décret d'une ces cités. ${ }^{59}$ Même s'il est vrai que ce composé a des emplois plus variés que le terme simple ou que $\delta \iota a ́ \sigma \kappa \in \psi l$, dans le contexte auquel nous nous référons, il apparaît comme synonyme des deux autres substantifs issus de la même racine. 
Ainsi, l'emploi d'un vocabulaire philosophique dans les scènes d'assemblées divines corrobore le rapprochement, qui est récurrent chez Lucien, entre dieux et faux philosophes.

Il existe encore bien d'autres exemples de ce parallèle, qui pourraient constituer à eux seuls un objet d'étude; les quelques cas que nous avons étudiés semblent néanmoins suffisants pour montrer que le recours ponctuel à un vocabulaire philosophique pour parler des dieux est tout à fait concerté et parfaitement cohérent avec le plan d'ensemble de Lucien, chez qui dieux et philosophes sont des objets récurrents de la satire - même si c'est à des degrés divers. En effet, dans la hiérarchie des charlatans, la palme revient incontestablement aux faux philosophes, et le simple fait de leur comparer les dieux est en soi, dans le contexte lucianesque, un procédé satirique puissant.

\section{Les dieux et leurs représentations: une relation problématique}

Lemploi de termes issus de la vie politique et de la philosophie tend à rapprocher les dieux du monde des hommes. Il est patent que, dans le monde que décrit Lucien, la référence n'est plus le divin, mais l'humain: c'est incontestablement un signe de déchéance, mais il y a pire encore. En effet, un degré supplémentaire est franchi dans la satire lorsque Lucien montre le rapport qu'entretiennent les dieux avec leurs représentations iconographiques.

Lorsqu'il s'agit, dans le Zeus tragédien, de déterminer l'ordre dans lequel les dieux doivent être assis pour assister à l'assemblée, Hermès et Zeus se trouvent dans le plus grand embarras; ils décident d'établir une hiérarchie entre les dieux en se fondant sur la matière dont est faite chaque statue de dieu (Zeus tragédien 7):

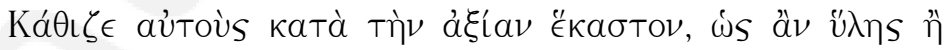

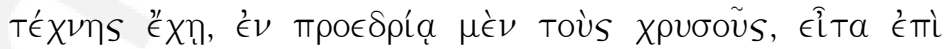

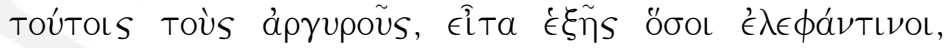

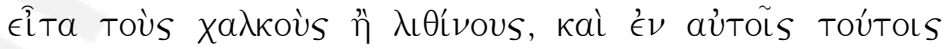

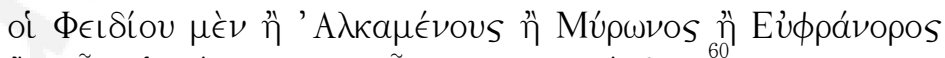

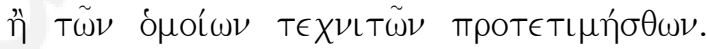

\footnotetext{
60 "Fais-les asseoir selon la valeur de chacun, c'est-à-dire d'après la matière ou l'art dont ils sont faits: au premier rang, ceux qui sont d'or, derrière eux, ceux qui sont d'argent, puis ceux qui sont d'ivoire, puis ceux de bronze ou de pierre. Parmi ces derniers, il faut donner la préséance à ceux qui sont de la main de Phidias, d'Alcamène, de Myron, d'Euphranor et d'autres artistes de semblable envergure".
} 
Ce n'est pas la qualité de citoyen, la mo入ıté́a, que l'on examine, mais l'ả $\xi i ́ a$ de chaque dieu, et cette dernière réside dans la valeur marchande des statues des dieux. En d'autres termes, ici, c'est la notion même de divinité qui est atteinte, car la notion de personne divine est remise en question: il n'existe plus un dieu, mais uniquement des images de ce dieu, et ces différentes images ne sont pas réductibles à une seule personne. Bien au contraire, elles se font concurrence! Ainsi, lorsqu'arrive dans l'assemblée des dieux l'Hermès de l'agora, c'est-à-dire la statue du dieu qui est placée sur l'agora (Zeus tragédien 33), Zeus s'adresse à lui d'une part comme à un être distinct d'Hermès, dont il differe par son corps couvert de poix et par son lieu de résidence (ce qui lui vaut d'être appelé Hermagoras dans le dialogue), mais, d'autre part, comme à quelqu'un qui est assimilable à Hermès - une sorte de double, un frère, que Zeus n'hésite pas à appeler "mon enfant", comme il le fait d'Hermès:

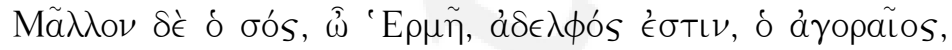

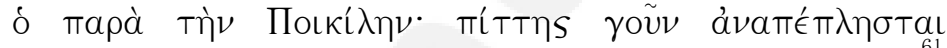

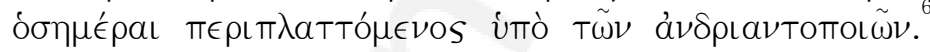

Si Hermagoras a une existence distincte de celle d'Hermès, c'est grâce au sculpteur qui l'a fait; le "corps" du dieu est le don gracieux de l'artisan. Il semble qu'on ait ici un écho d'une idée développée dans le Discours olympique de Dion de Pruse: celle que les grands sculpteurs ont une influence déterminante sur la représentation que les hommes se font des dieux; ainsi, Phidias, auteur de la célèbre statue de Zeus à Olympie, a changé la vision que les Grecs avaient du dieu, en créant une représentation qui s'est imposée et a supplanté toutes les autres. ${ }^{62} \mathrm{De}$ même, Lucien fait dire à Hermès qu'Aphrodite est devenue Aphrodite

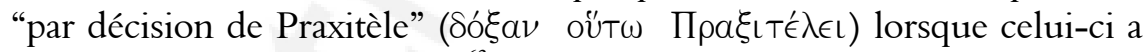
fait son Aphrodite de Cnide, ${ }^{63}$ mais sa remarque procède d'un point de

\footnotetext{
61 "Eh! Hermès, mais c'est ton frère, celui qui est sur l'agora, près du Pécile. Il est plein de poix, parce que les sculpteurs prennent son empreinte tous les jours".

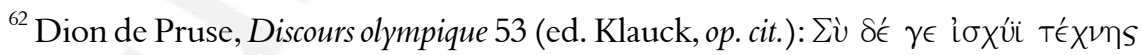

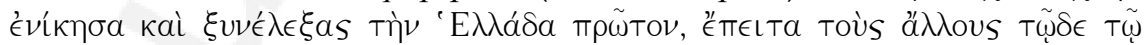

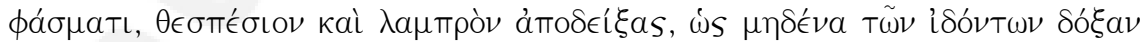

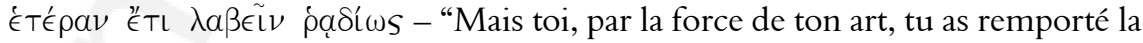
victoire et tu as rassemblé tout d'abord les Grecs, puis les autres grâce à cette représentation, en produisant une vision divine et claire, de sorte que nul, après l'avoir vue, ne put facilement s'en faire une autre idée". (traduit par nos soins)

${ }^{63}$ Zeus tragédien 10, cité infra, note 65.
} 
vue différent: elle ne traduit aucune admiration pour Praxitèle, mais uniquement la dépendance d'Aphrodite par rapport à celui-ci. Lucien souligne ainsi la subordination des dieux au bon vouloir du sculpteur et les conséquences inattendues qui en découlent: dans le cas d'Hermès, au lieu que le talent de l'artiste contribue à augmenter le respect pour le dieu, il rend celui-ci risible, car la statue est si réussie qu'on vient la copier quotidiennement, en laissant dessus des traces de poix qui montrent le peu de respect témoigné au dieu qu'elle représente. L'hommage rendu au talent du sculpteur se traduit par un outrage fait à la divinité, et comme celle-ci, contrairement aux dieux d'Homère, est impuissante à réagir, elle ne peut que subir son sort et faire rire d'elle.

Limportance d'un dieu dépend donc non de sa puissance, c'est-à-dire d'une qualité intrinsèque de la personne divine, mais de la valeur marchande de sa statue, liée à la matière dont elle est faite et au talent du sculpteur, autrement dit, d'un critère qui aboutit à nier sa nature divine et le ramène à une pure matérialité. Mais ce critère, si farfelu soit-il, s'avère encore insuffisamment précis: en effet, faut-il considérer la valeur propre au matériau dont la statue est faite, ou bien le coût réel de la statue? En d'autres termes, faut-il faire entrer en ligne de compte la quantité, ou bien ne considérer que la qualité de la matière? C'est le problème que le colosse de Rhodes, à l'effigie du dieu Hélios, pose à Hermès, car sa valeur marchande lui donne le droit d'avoir une bonne place à l'assemblée tandis que ses dimensions exceptionnelles l'empêchent d'y siéger (Zeus tragédien 11):

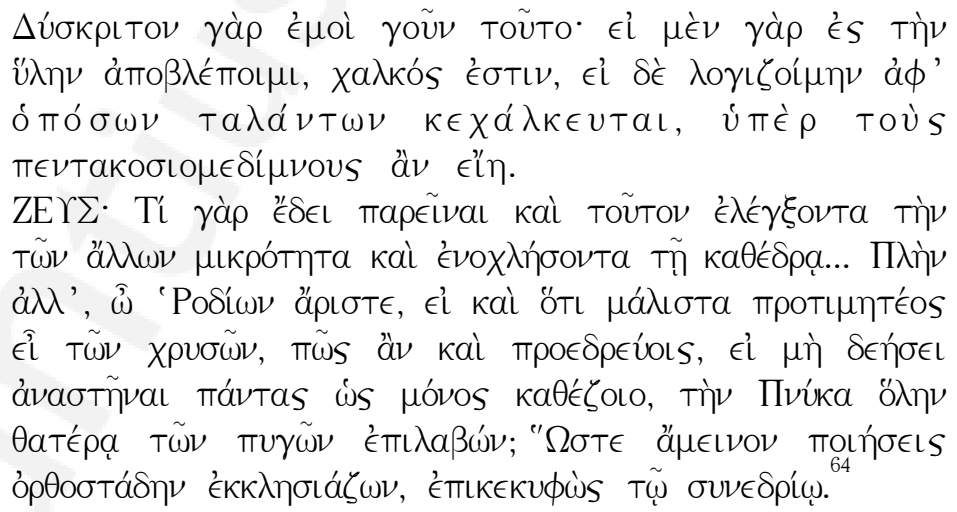

${ }^{64}$ "C'est un cas difficile à juger, au moins pour moi, car, si je considère la matière, il est de bronze, mais si je calculais combien de talents a coûté le bronze dont il est fait, il serait au-dessus des pentacosiomédimnes.

ZEUS: Quel besoin avait-il de venir, celui-là, pour mettre en évidence la petite taille des autres et obliger tout le monde à changer de place? Dis-moi, excellent 
Encore une fois, l'humain l'emporte sur le divin, dans la mesure où la valeur de chaque dieu est déterminée non par ses pouvoirs propres, mais par l'importance que les hommes lui accordent et dont témoigne la somme déboursée pour l'érection de la statue. Ce sont finalement les hommes qui donnent leur existence aux dieux et, au lieu qu'il y ait un dieu et de multiples représentations de celui-ci, chaque statue suffit à donner corps et existence à un être divin, de sorte que la personnalité divine est éclatée en une multitude d'individus.

La nouvelle hiérarchie ainsi établie entre dieux ne permet pas aux Olympiens d'affirmer leur suprématie, car ils se voient concurrencés par leurs confrères d'Asie (Zeus tragédien 8):

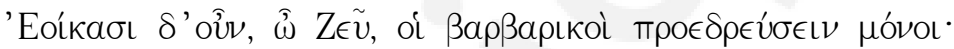

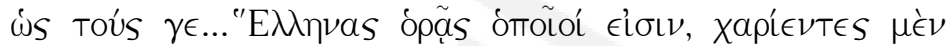

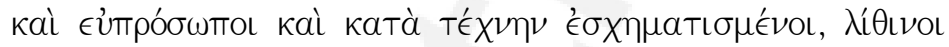

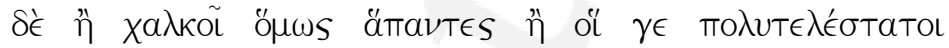

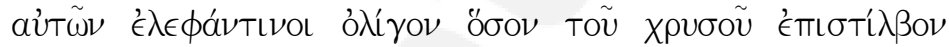

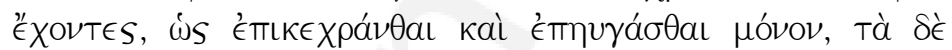

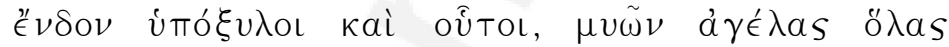

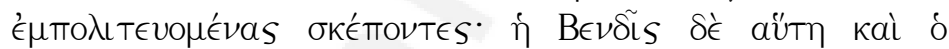

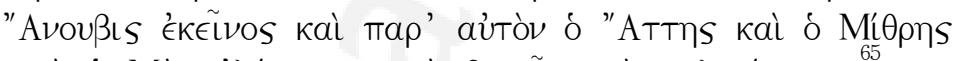

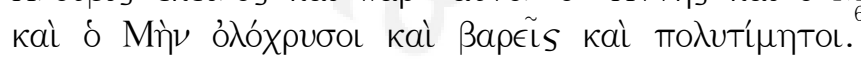

Même Aphrodite, qui tente de se faire valoir en jouant sur les mots et en rappelant qu'Homère la nomme "Aphrodite d'or", ${ }^{66}$ ne peut

Rhodien, même si tu dois avoir la préséance sur les dieux qui sont en or, comment pourrais-tu t'asseoir au premier rang, à moins d'obliger tous les assistants à se lever pour que tu puisses t'y asseoir seul? Tu occuperais toute la Pnyx avec une seule de tes fesses. Tu ferais mieux d'assister à la séance debout, penché sur l'assemblée”.

65 "On dirait bien, Zeus, que les dieux barbares vont occuper seuls les premiers rangs; car pour les Grecs, tu vois comme ils sont gracieux bien sûr, avec une belle figure, et dessinés avec art, mais ils sont tous de bronze ou de pierre; les plus luxueux d'entre eux sont d'ivoire agrémenté de quelques fioritures d'or, juste pour donner de la couleur et de la lumière; mais à l'intérieur, ils sont de bois eux aussi et abritent des colonies entières de souris. Au contraire, Bendis que voici et Anubis qui est là-bas, avec, à côté de lui, Attis, Mithra et Mèn sont d'or massif et d'un poids et d'un prix qui méritent vraiment considération".

${ }^{66}$ Cf.Zeus tragédien 10: Aphrodite: "Alors, prends-moi aussi, Hermès, et place-moi sur

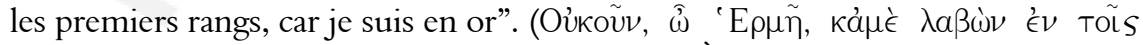

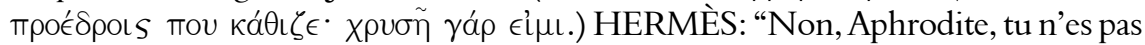
en or, du moins d'après ce que je peux voir. Mais, si je n'ai pas la berlue, tu as été 
accéder aux places d'honneur. Les dieux se prévalent de la matière dont ils sont faits extérieurement, comme pour mieux dissimuler leur vacuité intérieure. On peut reconnaître dans ce passage une inversion de l'image du silène, sous les traits duquel Alcibiade représente Socrate, dans le Banquet de Platon (216d-e). Alors que Socrate, comme le silène, ne révèle sa richesse que lorsqu'il est ouvert, les dieux illustrent la situation inverse: magnifiques à l'extérieur, ils ne sont que misère et désordre à l'intérieur, nouveau trait qui les rapproche des faux philosophes et les oppose à Socrate, qui est l'exemple à suivre: c'est lui que Lucien met ici en opposition avec ces prétendues divinités si ridicules, et ailleurs, avec les philosophes-charlatans, tous uniquement préoccupés de richesses et d'honneurs.

Le paradoxe cruellement et brillamment mis en relief par Lucien est que, de témoignage de piété qu'elles étaient à l'origine, ces statues ont fini par supplanter ceux qu'elles représentent: l'Olympe de Lucien est tellement surchargé de statues de dieux qu'il ne lui reste plus de place pour les dieux eux-mêmes, et sur ce point comme sur bien d'autres, Lucien est en phase avec la réalité de son temps: on sait que les grands sanctuaires étaient encombrés par les quantités d'offrandes, et notamment de statues, qui s'étaient accumulées dans les lieux sacrés au fil des siècles. Les statues des dieux occupent le premier plan, au sens propre comme au sens figuré, et finissent par cacher les dieux au lieu de les montrer.

Le procédé de Lucien constitue une sorte de tour de force car il inverse totalement le sens de cette accumulation de statues pour en faire un élément de la satire des dieux. À titre de comparaison, Dion de Pruse considérait comme intrinsèque à la nature humaine le besoin de représenter ses dieux, en comparant les hommes à des enfants qui seraient privés de leurs parents et tendraient les mains vers eux, dans leur désir de les toucher; c'est un tel manque que viendrait combler la représentation

taillée, je crois, dans la pierre blanche du Pentélique; puis, sur la décision de Praxitèle,

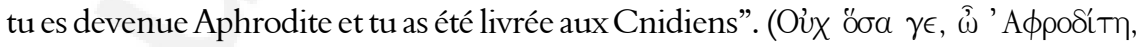

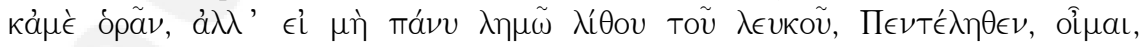

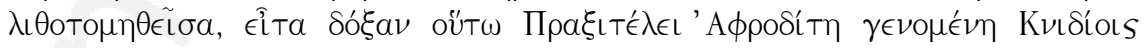
тарєSóOn s.) APHRODITE: "Cependant je te citerai un témoin digne de foi, Homère, qui dit d'un bout à l'autre de ses rhapsodies que moi, Aphrodite, je suis d'or". (Kaì

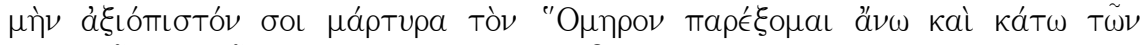

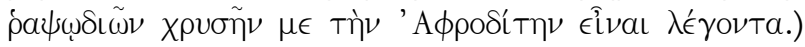


anthropomorphique des dieux grecs. ${ }^{67}$ Lucien, au contraire, détourne son regard de l'humain pour se porter sur le divin et, loin de fustiger les hommes pour leurs faiblesses, tourne en dérision la divinité, qui n'est rien d'autre qu'une vue de l'esprit créée par les hommes.

Il est une tradition que Lucien contribue puissamment à illustrer, celle du spoudogeloion, que l'on peut définir comme un procédé consistant à produire un texte d'apparence comique mais qui cache en lui une vérité d'ordre "sérieux".

Lucien est sérieux dans la satire, et notamment dans la satire des dieux: celle-ci ne se résume pas à une moquerie ponctuelle dictée par l'envie de mettre les rieurs de son côté et destinée à s'assurer un succès à bon compte, même si, bien sûr, Lucien devait avoir aussi ce désir. On a vu en effet que la satire qu'il fait des dieux est structurée, cohérente et qu'elle a des liens importants avec d'autres thèmes qui lui sont également chers: la religion est un avatar de la charlatanerie dont bon nombre d'humains trop crédules sont les victimes.

Comme le souligne Maurice Croiset, ${ }^{69}$ Lucien n'a pas pour objectif de convaincre ses auditeurs, mais de leur mettre sous les yeux la faiblesse des arguments en faveur de la religion. Plutôt qu'à une satire des dieux, Lucien se livre à une satire de la représentation des dieux par les hommes, procédé qui évite une condamnation brutale sur un sujet que notre auteur n'estime peut-être pas assez grave pour cela. Le ton employé à l'égard des dieux est en effet notablement moins acerbe que celui qui est réservé aux faux philosophes. La religion et ses

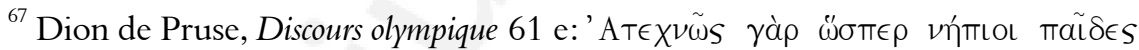

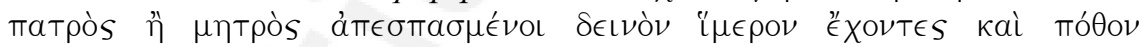

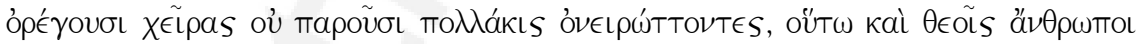

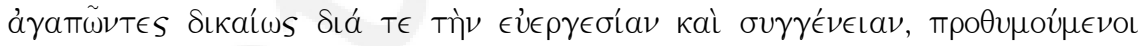

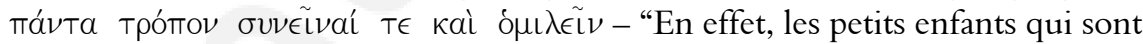
arrachés à leur père ou à leur mère éprouvent un désir irrépressible et, bien des fois, tendent leurs bras vers eux alors qu'ils ne sont pas là, lorsqu'ils les voient en rêve; c'est exactement ainsi que les humains se comportent vis-à-vis des dieux, les aimant à juste titre, eu égard à leurs bienfaits et à leur parenté avec eux et en s'efforçant par tous les moyens d'être en leur présence et de s'entretenir avec eux".

${ }^{68}$ Cf. Camerotto, op. cit., p. 127: "Lo spoudogeloion è proprio del testo che appare comico, ma che nasconde e poi rivela inaspettata una verità, secondo la formula oraziana del ridentem dicere uerum o la definizione che Diogene Laerzio dà delle

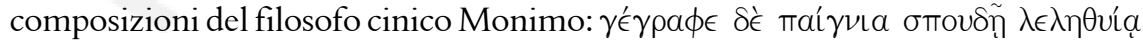
$\mu \epsilon \mu \iota \gamma \mu \dot{\epsilon}^{\prime} \alpha^{\prime}$.

${ }^{69}$ Cf. Croiset, op. cit., p. 211.
} 
oripeaux font partie des faiblesses humaines face auxquelles l'indulgence est de mise et le rire, sans doute plus approprié que la polémique. À cet égard, le jugement le plus ferme, aux accents nettement épicuriens - se trouve sans doute dans l'ouverture du traité Sur les sacrifices:

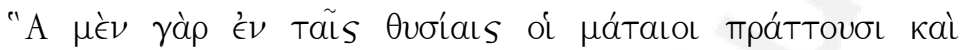

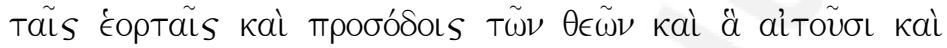

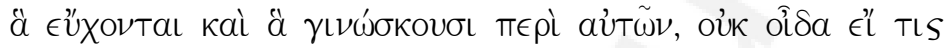

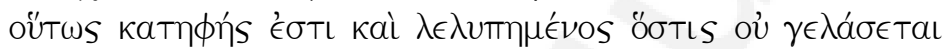

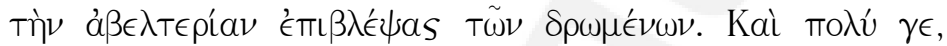
ổ

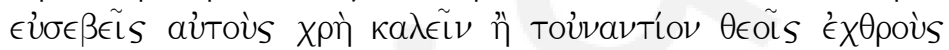

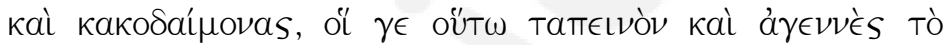

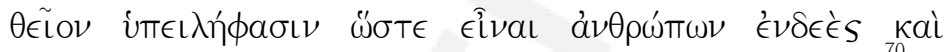

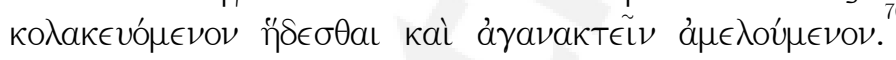

\section{Références}

BOMPAIRE, J. Lucien écrivain, imitation et création. Paris: De Boccard, 1958.

CAMEROTTO, A. Le metamorfosi della parola: studi sulla parodia in Luciano di Samosata. Pise/ Rome: Istituti editoriali e poligrafici internazionali, 1998.

CASTER, M. Lucien et la pensée religieuse de son temps. Paris: Les Belles Lettres, 1937. CROISET, M. Essai sur la vie et les ouvres de Lucien. Paris: Hachette,1882.

DELZ, J. Lukians Kenntnis der athenischen Antiquitäten. Fribourg: Paulus-druckerei, 1950. DUBEL, S. Dialogue et autoportrait: les masques de Lucien. In: BILLAULT, A. (org.). Lucien de Samosate. Paris: De Boccard, 1994, p. 19-26.

FOLLET, S. Lettre de Marc-Aurèle aux Athéniens (EM 13366): nouvelles lectures et interprétations. Revue de philologie, de littérature et d'histoire anciennes, Paris, vol. 105, p. 29-43, 1979.

\footnotetext{
${ }^{70}$ Sur les sacrifices 1: "À voir ce que font les imbéciles dans les sacrifices, les fêtes et les processions en l'honneur des dieux, les requêtes et les prières qu'ils leur adressent ainsi que l'idée qu'ils se font d'eux, je ne sais pas si quelqu'un peut être assez sombre et affligé pour ne pas rire en voyant la stupidité de leur conduite. Et je pense que, bien avant de rire, cette personne se demandera s'ils méritent le qualificatif de 'pieux' ou, au contraire, ceux de 'ennemis des dieux' et de 'malheureux', puisqu'ils considèrent que la divinité est basse et vulgaire au point d'avoir besoin des hommes, d'aimer être flattée par eux et de se fâcher si on la néglige”.
} 
FREYBURGER, M. L.; FREYBURGER, G.; TAUTIL, J. C. Sectes religieuses en Grèce et à Rome. Paris: Les Belles Lettres, 1986.

GAUTHIER, P. Symbola: les étrangers et la justice dans les cités grecques. Nancy: Annales de l'Est, 1972.

GRAINDOR, P. Un milliardaire antique: Hérode Atticus et sa famille. Le Caire: Misr, 1930.

HOUSEHOLDER, F. W. The mock decrees in Lucien. TAPhA, Baltimore, vol. 71, p. 199-216, 1940.

JONES, C. P. Culture and society in Lucian. Cambridge MA: Harvard University Press, 1986.

KARAVAS, O. NHFE KAI MEMHNSO APISTEIN (Hermot. 47): la religiosité de Lucien. In: BARTLEY, A. (org.). A Lucian for our times. Cambridge: Cambridge Scholars Publishing, 2009, p. 137-144.

KLAUCK, H.J. Dio von Prusa, Olympikos. Darmstadt: Wissenschaftliche Buchgesellschaft, 2000.

LIGHTFOOT, J. L. Lucian: On the Syrian goddess. Oxford: Oxford University Press, 2003.

OLIVER, J. H. Marcus Aurelius: aspects of civic and cultural policy in the East. Hesperia. Princeton, supplement XIII, 1970.

OLIVER, J. H. The actuality of Lucian's "Assembly of the Gods". American Journal of Philology, Baltimore, vol. 101, p. 303-313, 1980.

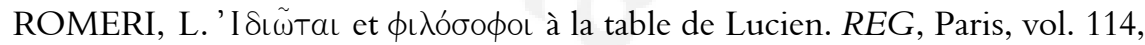
p. 647-655, 2001.

SCHWARTZ, J. Biographie de Lucien de Samosate. Collection "Latomus" LXXXIII. Bruxelles: Latomus, 1965.

TOBIN, J. Herodes Atticus and the city of Athens: patronage and conflict under the Antonines. Amsterdam: J. C. Gieben, 1997. 